\title{
APLICACIÓN DEL MÉTODO SERVQUAL EN LOS SERVICIOS DE ALUMBRADO PÚBLICO DE ZAPOPAN, JALISCO
}

\author{
AUTORES: Mtro. Luis Joel Torres Arreola \\ Dr. Ernesto Raúl González Ramírez \\ Universidad de Guadalajara \\ Centro Universitario de Ciencias Económico Administrativas \\ División de Gestión Empresarial \\ Departamento de Mercadotecnia y Negocios Internacionales
}

\author{
Dirección: \\ Periférico Norte 799 \\ Núcleo Universitario Los Belenes C. P: 45100 \\ Zapopan, Jalisco, México \\ Tel: $\mathbf{3 7 7 0 3 3 3 2}$ \\ Correo: luisjtorres@hotmail.com \\ eraulg@cucea.udg.mx
}

\section{RESUMEN}

Considerando que el uso de los Sistemas de Calidad en el sector público Mexicano se encuentra en un proceso inicial, este estudio revisa solo un aspecto de la implementación de los Sistemas de Calidad. En este caso, el Sistema de Calidad esta identificado como la certificación de las normas de calidad de la Organización Internacional de Estandarización (ISO, en inglés).

El objetivo de esta investigación es medir a través del modelo SERVQUAL el grado en que el personal de la Dirección de Alumbrado Público de Zapopan (D. A. P. Z), Estado de Jalisco, ha asumido una filosofía de calidad con respecto a los servicios que ofrecen a través de su estructura organizacional, tratándose en este caso de una investigación aplicada. Es importante considerar que esta área tiene tres años de haber implementado un sistema de calidad como lo es la certificación ISO 9002.

\section{Introducción.}

El desarrollo de la administración de la Calidad Total (TQM, en inglés) en el sector público ha tenido un problema existencial. Palabras tales como "clientes" y "rentabilidad" no se han aceptado rápidamente en el servicio público que cuenta con una historia de autoridad centralizada y de procedimientos burocráticos, este legado cultural difiere con el concepto de propiedad individual o iniciativa, conceptos fuera de contexto para la mayoría de los servidores públicos. El concepto de servicio al cliente y calidad total, los nuevos imperativos, parecerán particularmente fuera de lo habitual en el sector público y esto hace que el proceso de establecimiento de la Calidad Total sea extenso y complicado (Moon et al, 1998, p. 1).

Las organizaciones del sector público no deben ser inmunes a la presión que esta sujeta una organización privada para producir servicios de calidad para brindar satisfacción a los clientes. Los servicios en el sector público están experimentando presiones como resultado de las limitaciones financieras, vigilancia por parte de los tres poderes del Estado y la tensión política que vive el país. Estos fenómenos producen un deseo por la eficientación y aumento de la calidad en los servicios públicos.

Durante los años noventa, el sector público en los Estados Unidos y en el Reino Unido comenzaron la implementación de modelos de calidad en algunas agencias federales y estatales con el propósito de mejorar los servicios y reducir los gastos de cada agencia. Algunos estudios mostraron las dificultades para la adaptación del modelo de 
Calidad Total en el sector público, de igual forma se mostró que existía un alto nivel de fracasos en la implementación de este modelo, estos estudios argumentaron que este alto grado de fracasos en la implementación se debió a la falta de un modelo especifico para la implementación de la Calidad Total en el sector público (Hyde, 1992, p.23).

La introducción de la administración de Calidad Total (TQM) al sector público tiende a la resistencia, y la pregunta de hasta qué punto la administración de la Calidad Total o Sistemas de Calidad pueden adaptarse a los requisitos del sector público, o si hay complejidades más profundas acerca de la naturaleza cambiante de relaciones laborales, deben ser abordadas durante la toma de decisión en aceptar o no la implementación de un Sistema de Calidad.

Los Sistemas de Calidad son una manera de manejar y mejorar la efectividad, flexibilidad y competitividad de una organización en su conjunto; esto significa que la adopción de este modelo es la aceptación de un cambio orgánico y una nueva cultura organizacional. La adopción de la Calidad Total o de un Sistema de Calidad puede mejorar la calidad de los servicios gubernamentales. El empleo de este acercamiento puede ser considerado una importante aportación a la eficientación del sector público en México.

Considerando que el uso de los Sistemas de Calidad en el sector público Mexicano esta en un proceso inicial, este estudio revisa solo un aspecto de la implementación de los Sistemas de Calidad. En este caso, el Sistema de Calidad esta identificado como la certificación de las normas de calidad de la Organización Internacional de Estandarización (International Standardization Organization, en inglés).

El objetivo de esta investigación es medir el grado en que el personal de la Dirección de Alumbrado Público de Zapopan (D. A. P. Z), Estado de Jalisco, ha asumido una filosofía de calidad con respecto a los servicios que ofrecen a través de su estructura organizacional, tratándose en este caso de una investigación aplicada. De forma concreta los aspectos medidos en este estudio serán las percepciones del personal con respecto al nivel de importancia que le atribuyen a las dimensiones de la calidad en los servicios (elementos tangibles, fiabilidad, capacidad de respuesta, seguridad y empatia) y a demás la medición de las deficiencias que el personal percibe en la realización del servicio (estas mediciones forman parte del modelo SERVQUAL). Es importante considerar que esta área tiene tres años de haber implementado un sistema de calidad como lo es la certificación ISO 9002.

La estructura del presente trabajo se divide en la revisión teórica de la calidad total en el servicio, en el diseño metodológico utilizado en esta investigación, los resultados obtenidos, y finalmente las conclusiones y recomendaciones sobre esta investigación.

\section{Desarrollo.}

\section{Modelos de Calidad Total en las Organizaciones Prestadoras de Servicios.}

Con respecto a los modelos teóricos sobre la calidad total, se han encontrado varias aportaciones las cuales resaltan las dimensiones a través de las que se debe medir el nivel de calidad bajo la perspectiva del enfoque de la calidad total.

A partir de estas dimensiones la organización evalúa de forma sistémica el estado actual de la misma, para, una vez, detectadas las deficiencias en esas áreas proponer mejoras. Dentro de los autores que realizan aportaciones en este sentido, se mencionan a Sureschchandar, Rajendran, Chandrasekharan, y Anantharaman, y Zeithaml, Parasuraman y Berry.

En el desarrollo teórico del presente trabajo se especifican los contenidos de estos dos grupos de autores, seleccionando para la aplicación empírica en el servicio de alumbrado público en el Municipio de Zapopan, al modelo de Zeithaml, Parasuraman y Berry. 
Revista Eletrônica de Ciência Administrativa (RECADM) - ISSN 1677-7387

Faculdade Cenecista de Campo Largo - Coordenação do Curso de Administração

v. 2, n. 1, maio/2003 - http://revistas.facecla.com.br/index.php/recadm/

El modelo propuesto por Sureschchandar, Rajendran, Chandrasekharan, y Anantharaman (2001) proporcionan una investigación de la literatura respecto a la Calidad Total en los Servicios (Total Quality Service, en inglés) identificando 12 dimensiones de la administración de calidad consideradas muy importantes para la institución de un modelo de Calidad Total en organizaciones de servicio. Las dimensiones que se han identificado son las siguientes: (1) El compromiso de la alta dirección y un liderazgo visionario. (2) Administración de los recursos humanos, (3) Sistema técnico, (4) Sistemas de información y análisis, (5) Benchmarking, (6) Mejora continua, (7) El enfoque hacia el cliente, (8) La satisfacción del personal, (9) La intervención de los sindicatos, (10) La responsabilidad social, (11) Apariencia física de los servicios, Finalmente, (12) La cultura de servicio. Estas dimensiones pueden agruparse ampliamente bajo tres categorías (Sureschchandar, Rajendran, Chandrasekharan, y Anantharaman, 2001, p. 9).

El trabajo presentado por estos autores muestra un modelo conceptual para la administración de la Calidad Total en los Servicios que sugiere que los 12 factores propuestos anteriormente son críticos para el establecimiento de un ambiente de Calidad Total en organizaciones prestadoras de servicio. Estas dimensiones tienen funciones diferentes para desarrollarse en un modelo de Calidad Total en los Servicios.

El compromiso de la alta dirección actúa como la guía de un modelo de esta naturaleza. Este compromiso aunado con un liderazgo visionario operará en el sistema organizacional que consiste en subsistemas como la administración de los recursos humanos, el enfoque hacia el cliente, el sistema técnico, sistemas de información y comunicación, elementos tangibles y responsabilidad corporativa. Los asuntos de las relaciones industriales son representados por la intervención de los sindicatos y el estándar de comparación por medio del benchmarking.

El advenimiento de la tecnología al servicio del cliente es intenso y está en pleno proceso de crecimiento. Pero, en general, la mayoría de las organizaciones enfocadas al servicio brindan esta prestación a través de su personal que forma la interacción entre los clientes y las organizaciones. Por consiguiente, el enfoque hacia el cliente y la satisfacción del personal son concebidas como las principales metas del modelo de la Calidad Total en los Servicios (Sureschchandar, Rajendran, Chandrasekharan, y Anantharaman, 2001, p. 10).

Como segundo modelo (aplicación para este trabajo) para Zeithaml, Parasuraman, y Berry (1993), la información contenida en la bibliografía existente durante la década de los noventa sobre calidad no era suficiente para desarrollar una base conceptual global que facilite la comprensión y el perfeccionamiento de la calidad de los servicios, ya que esta era predominantemente orientada hacia los productos tangibles. Basados en la definición conceptual de la calidad del servicio y en los diez criterios valorativos que los autores encontraron en la investigación exploratoria, se embarcaron en la fase cuantitativa de la investigación con el propósito de desarrollar un instrumento que permitiese medir las percepciones de los usuarios sobre la calidad de los servicios.

Estas investigaciones arrojaron cinco criterios o dimensiones de calidad en el servicio. Estos cinco criterios diferenciados incluyen todas las facetas de los diez criterios que originalmente los autores habían conceptualizado. Estos criterios son: elementos tangibles, fiabilidad, capacidad de respuesta, seguridad y empatia (Zeithaml, Parasuraman, y Berry, 1993, p.28).

Los hallazgos encontrados en este estudio, fueron parte de una investigación con directivos de empresas importantes de servicios en los Estados Unidos de Norteamérica, estos individuos procedían de departamentos como el de mercadotecnia, operaciones, y atención al cliente. Entre los servicios que brindan estas empresas se encuentran la banca minorista, servicios de tarjetas de crédito, corretajes de valores, y reparación y mantenimiento de aparatos. Estas investigaciones revelaron cuatro factores clave causantes de las deficiencias internas que contribuyen a los bajos niveles de calidad en el 
servicio percibido por los clientes; es decir: Deficiencia 1, la discrepancia entre las expectativas del cliente y las percepciones que tienen los directivos sobre esas expectativas; Deficiencia 2, la discrepancia ente la percepción de los directivos sobre las expectativas de los clientes y las especificaciones o normas de calidad del servicio; Deficiencia 3, la discrepancia entre las normas de calidad del servicio y el servicio real ofrecido; y Deficiencia 4, la discrepancia entre el servicio real y lo que se comunica a los clientes sobre él. Se vincularon las percepciones del cliente sobre la baja calidad del servicio (Deficiencia 5) con esas cuatro deficiencias con el fin de estructurar un modelo conceptual de la calidad del servicio. Este modelo conceptual sirve como marco general que, de forma esquemática, permite la comprensión, medición y perfeccionamiento de la calidad del servicio (Zeithaml, Parasuraman, y Berry, 1993, p. 56).

EI modelo SERVQUAL, es un instrumento resumido de escala múltiple, con un alto nivel de fiabilidad y validez, que las organizaciones pueden usar para comprender mejor las expectativas y percepciones que los clientes tienen con respecto a un servicio. Este instrumento puede aplicarse a una amplia gama de servicios. SERVQUAL suministra un esquema o armazón básico basado en un formato de representación de las expectativas y percepciones que incluye declaraciones para cada uno de los cinco criterios de la calidad en el servicio (elementos tangibles, fiabilidad, capacidad de respuesta, seguridad y empatía). Cuando es considerado necesario, este esquema se puede adaptar o complementar agregando algunas características específicas que respondan a las necesidades de la investigación (Zeithaml, Parasuraman, y Berry, 1993, p.205).

Una organización no sólo puede evaluar la calidad global de su servicio, tal y como la perciben los clientes, sino que, además puede determinar cuáles son los criterios y las facetas clave, con el propósito de centrar en esas áreas los esfuerzos que realice para mejorar la calidad de sus servicios. El instrumento SERVQUAL puede aplicarse de maneras muy diferentes: a) para comparar las expectativas y las percepciones de los clientes a lo largo del tiempo, b) para comparar las puntuaciones de SERVQUAL de una compañía contra las puntuaciones de los competidores, c) para examinar los segmentos de clientes que poseen percepciones diferentes sobre la calidad, y por último, (la aplicación utilizada en este estudio) d) evaluar las percepciones de los clientes internos sobre la calidad (Zeithaml, Parasuraman, y Berry, 1993, p.207).

\section{Metodología.}

En el párrafo anterior se determino que el modelo a utilizar para él diagnostico y propuestas de mejora para la calidad del servicio en la Dirección de Alumbrado Público de Zapopan (D. A. P. Z.) es el modelo SERVQUAL. La metodología utilizada al respecto fue la que se describe a continuación. (Ver Figura 1, p. 11).

Como sujetos de estudio se tomó el nivel municipal de gobierno porque comparado con los gobiernos federales y estatales, el gobierno municipal proporciona primordialmente los servicios básicos a la ciudadanía, por ejemplo: Servicio Público de Limpieza, Servicio de Alumbrado Público, el Servicio de Transporte Urbano, Servicio de Seguridad, etc. De acuerdo a su progreso en la implementación de la filosofía de la calidad, prontitud de información, y accesibilidad geográfica los investigadores decidieron que la Dirección de Alumbrado Público de Zapopan (D. A. P. Z) era el más conveniente para este estudio. 
Revista Eletrônica de Ciência Administrativa (RECADM) - ISSN 1677-7387

Faculdade Cenecista de Campo Largo - Coordenação do Curso de Administração

v. 2, n. 1, maio/2003 - http://revistas.facecla.com.br/index.php/recadm/

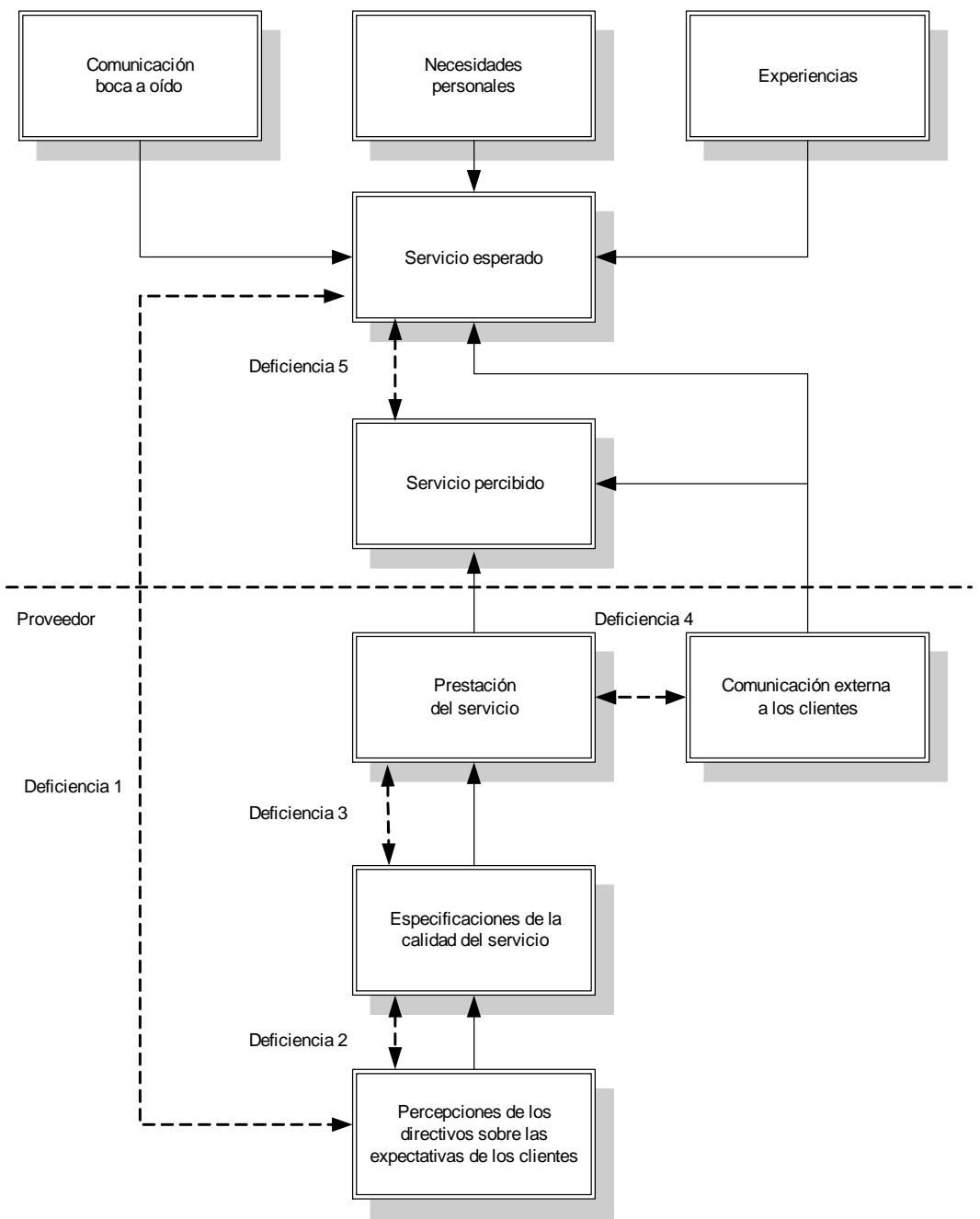

El marco de la muestra para este estudio está compuesto por las personas que trabajan en la Dirección de Alumbrado Público de Zapopan en la municipalidad de Zapopan, Estado de Jalisco. De acuerdo con la estratificación realizada para la obtención de la muestra se entrevistaron 32 empleados incluyendo el nivel directivo (ver Tabla 1). La Dirección de Alumbrado Público de Zapopan cuenta con 70 empleados. De acuerdo con su organigrama el personal esta dividido en siete áreas: Dirección: 2 empleados; Área operativa: 43 empleados; Administración y Área de Control de Calidad: 3 empleados; Área Técnica o Estudios y Proyectos: 5 empleados; Atención Ciudadana: 4 empleados, Almacén: 10 empleados; y Control y Uso Eficaz de Energía: 3 empleados.

Tabla 1. Descripción de la muestra estratificada

\begin{tabular}{|c|c|c|c|c|}
\hline Estratos & Población & $\%$ & Enc $\%$ & Muestra \\
\hline 1 & 43 & $61.43 \%$ & 18 & 10 \\
\hline 2 & 4 & $5.71 \%$ & 2 & 4 \\
\hline 3 & 5 & $7.14 \%$ & 2 & 4 \\
\hline 4 & 4 & $5.71 \%$ & 2 & 4 \\
\hline 5 & 10 & $14.30 \%$ & 4 & 6 \\
\hline 6 & 4 & $5.71 \%$ & 2 & 4 \\
\hline Total & 70 & $100.00 \%$ & 30 & 32 \\
\hline
\end{tabular}

Para la obtención de la información era importante tener una estrategia para la determinación de las preguntas. De acuerdo con Zeithaml, Parasuraman, \& Berry (1993), 
Revista Eletrônica de Ciência Administrativa (RECADM) - ISSN 1677-7387

Faculdade Cenecista de Campo Largo - Coordenação do Curso de Administração

v. 2, n. 1, maio/2003 - http://revistas.facecla.com.br/index.php/recadm/

el modelo SERVQUAL puede adaptarse y agregar características específicas que responden a las necesidades de la investigación. Puesto que el modelo de SERVQUAL es un instrumento concebido en organizaciones privadas, los investigadores hicieron la selección y adaptación de las declaraciones que podrían usarse en el estudio de una organización pública de servicios (Zeithaml, Parasuraman, y Berry, 1993, p. 205). Para adaptar el instrumento de medición SERVQUAL, los investigadores seleccionaron las declaraciones que podrían adaptarse en una organización pública de servicio con un sistema de administración de calidad, tales declaraciones giraron alrededor de la percepción sobre los medios físicos, la información visible de los servicios, el servicio rápido, el conocimiento necesario para responder preguntas, la atención individualizada, la comprensión de las necesidades específicas del ciudadano, el trabajo en equipo, la vigilancia y los sistemas del mando, las comunicaciones horizontales, los niveles jerárquicos, el compromiso de la dirección entre otros los cuales se plasman en el contenido- posterior. Estas características se encuentran en una organización pública de servicios y deben considerarse para medición de la filosofía de la calidad total para, en función de ello, trabajar en base a este enfoque.

Finalmente, del modelo general de SERVQUAL, fueron utilizados aquellos componentes que permiten evaluar la perspectiva interna del personal con respecto a las 5 dimensiones de calidad del servicio y cómo se comportan en la organización o servicio objeto de estudio, además de ofrecer qué nivel de importancia le otorgan a cada una de estas dimensiones. Colateralmente el personal interno tuvo la oportunidad de emitir su juicio sobre los aspectos del desempeño con respecto a las actuaciones relativas a las operaciones internas de la organización. Con esto y las valoraciones anteriores se definió cómo está preparada la D. A. P. Z. con respecto a la filosofía de la calidad total. En el cuestionario utilizado para obtener las valoraciones se precisan los aspectos considerados en el estudio, a partir de los cuales, se describe el fenómeno estudiado. Más concretamente, las cinco dimensiones evaluadas fueron los elementos tangibles, fiabilidad, capacidad de respuesta, seguridad y empatia.

Las declaraciones no incluidas en este estudio fueron las relacionadas con ventas, competidores y la porción del mercado, estas claramente identificadas como características de una organización privada.

\section{Análisis de las Fiabilidad y Validez de Instrumentos de Medición.}

El estudio de la fiabilidad de las escalas utilizadas en la encuesta es un paso necesario en este trabajo. La fiabilidad de una escala se utiliza para comprobar su consistencia interna ${ }^{1}$.

\footnotetext{
1 Cada variable o ítem es evaluado con el resto para determinar si los resultados obtenidos son estables y responden realmente a lo que queremos medir.

El coeficiente alpha de Cronbach y las correlaciones ítem-total son los indicadores adecuados para el análisis. El primero, expresa la relación entre el cuestionario utilizado y el resto del cuestionario que contengan el mismo número de ítems y que pueden ser construidos dentro de un mismo universo hipotético de ítems que midan la característica de interés. Este índice muestra la correlación entre la puntuación que una persona obtiene en el cuestionario actual y la que hubiese obtenido de habérsele preguntado todo el universo de ítems posible.

El coeficiente alpha de Cronbach es adecuado cuando obtiene valores superiores a 0.7 . Las correlaciones ítem-total se determinan a través del coeficiente de correlación de Pearson (R2), el cual puede oscilar entre menos uno y más uno y es el resultado del cociente entre la covarianza de las variables y sus desviaciones típicas. Y el coeficiente de correlación múltiple, que se obtiene al tomar el ítem de interés como una variable dependiente y el resto de los ítems considerados como variables independientes. Ambos miden el grado de asociación entre las variables. Las escalas del estudio sujetas a la valoración alpha fueron los correspondientes a las preguntas 1, 5 y 6 del cuestionario.

De acuerdo con los valores alpha obtenidos en este estudio, en la primera escala dirigida a medir las percepciones del personal interno sobre lo que espera la ciudadanía de la calidad en el servicio en cuanto a las dimensiones (pregunta 1) se obtuvo el valor alpha de 0.7932. Para las escalas relativas a los
} 
Para todos los ítems de cada una de esas medidas se utilizó una escala de $\mathbf{1}$ a 10 posiciones. A este respecto los valores de 1 a 4 se interpretan como total desacuerdo, 5 y 6 como indiferente, y los contenidos de 7 a 10 como total acuerdo.

Para la medición del nivel de la importancia atribuida a las dimensiones del servicio que ofrece la Dirección de Alumbrado Público para los ciudadanos según el personal cuando se evalúa la calidad de servicio de la D. A. P. Z. se tuvo en consideración cinco dimensiones o características que son la apariencia, la habilidad, la disposición, el conocimiento y trato amable, y el cuidado y atención individualizada. Para ello se optó por la variante de reparto de $\mathbf{1 0 0}$ puntos entre todas, dando el mayor puntaje a la característica más importante y así sucesivamente hasta que el total del puntaje se cierre en cien puntos. Después de valorar las cinco características señaladas previamente se eligen las tres características más importantes. Empezando por la más importante, seguido por la segunda más importante, y finalmente la tercera más importante.

\section{Resultados.}

1. Percepciones del personal interno sobre lo que espera la ciudadanía de la Dirección de Alumbrado Público (pregunta 1).

Los resultados globales arrojan la identificación de los antecedentes potenciales que provocan las deficiencias en la calidad en el servicio. Respecto a las expectativas del personal interno sobre lo que espera la ciudadanía de la Dirección de Alumbrado Público, se encontró que existe una aceptable orientación a la investigación de marketing (entendida como orientación hacia el mercado, en este caso, la ciudadanía), es decir, la Dirección de Alumbrado Público le da importancia a la apariencia de los elementos tangibles tales como el equipo, instalaciones, información visible y una buena apariencia del personal. Respecto a la comunicación ascendente que debe ofrecer una organización de servicios encontramos que el personal de esta área considera que es importante cumplir las promesas de servicio, mostrar un sincero interés en solucionarlo y realizar el servicio bien a la primera vez. Estos antecedentes potenciales muestran que el personal esta relacionado con términos utilizados en la aplicación de la filosofía de la calidad total (ver Tabla 2).

Para determinar el grado en que la discrepancia entre la percepción de los directivos sobre las expectativas de los clientes y las especificaciones o normas de calidad del servicio afectan la calidad se encontró que el compromiso de la dirección con la calidad en el servicio es aceptable así como el establecimiento de objetivos y normas estándar (ver Tabla 2).

Tabla 2: Valoración de percepciones

\begin{tabular}{|c|c|c|c|}
\hline & $\mathbf{N}$ & Media & Desv. Stad. \\
\hline 1. Equipos de apariencia moderna & 27 & 7.74 & 2.97 \\
\hline 2. Instalaciones físicas & 27 & 9.78 & 1.15 \\
\hline 3. Buena apariencia & 27 & 8.74 & 2.57 \\
\hline 4. Ofrecer información visible & 27 & 8.96 & 1.74 \\
\hline $\begin{array}{l}\text { 5. Promete hacer un servicio en un } \\
\text { tiempo }\end{array}$ & 27 & 8.96 & 2.52 \\
\hline 6. Sincero interés en solucionarlo & 27 & 9.30 & 1.96 \\
\hline
\end{tabular}

aspectos organizacionales de la prestación del servicio y la contribución del personal a la calidad en el servicio (pregunta 5 y 6 ), los valores alpha obtenidos no son muy exitosos 0.6533 y 0.6928 respectivamente, pero como éstos difieren muy poco de 0.7 se decidió contemplarlos como parte del estudio. 
Revista Eletrônica de Ciência Administrativa (RECADM) - ISSN 1677-7387

Faculdade Cenecista de Campo Largo - Coordenação do Curso de Administração

v. 2, n. 1, maio/2003 - http://revistas.facecla.com.br/index.php/recadm/

\begin{tabular}{|c|c|c|c|}
\hline $\begin{array}{l}\text { 7. Realizar bien el servicio en el } \\
\text { primer contacto }\end{array}$ & 27 & 9.48 & .85 \\
\hline 8. Libres de errores & 27 & 7.85 & 3.01 \\
\hline $\begin{array}{l}\text { 9. Comunicar cuando concluirá el } \\
\text { servicio }\end{array}$ & 27 & 8.48 & 2.62 \\
\hline 10. Servicio rápido a sus clientes & 27 & 8.56 & 2.06 \\
\hline 11. Dispuesto siempre a ayudar & 27 & 8.67 & 2.56 \\
\hline 12. Nunca estar demasiado ocupado & 27 & 7.96 & 2.86 \\
\hline 13. Transmitir confianza & 27 & 9.78 & .64 \\
\hline 14. Siempre amable & 27 & 9.96 & .19 \\
\hline 15. Tener conocimientos suficientes & 27 & 9.56 & 1.05 \\
\hline 16. Atención individualizada & 27 & 7.63 & 2.92 \\
\hline $\begin{array}{l}\text { 17. Orientar sobre todos los } \\
\text { servicios }\end{array}$ & 27 & 8.59 & 2.36 \\
\hline $\begin{array}{l}\text { 18. Comprender las necesidades } \\
\text { específicas }\end{array}$ & 27 & 8.96 & 1.91 \\
\hline 19. Tener buena reputación & 27 & 9.78 & .80 \\
\hline
\end{tabular}

\section{Importancia de las dimensiones de la Calidad en el Servicio (pregunta 2).}

Los autores Zeithaml, Parasuraman y Berry (1993) identifican tres facetas fundamentales que definen las diferencias que existen entre los servicios y los bienes físicos y que se refieren a la forma en que son producidos, en que son consumidos y en que son evaluados. Primero, los servicios son básicamente intangibles. Ya que son prestaciones y experiencias más que objetos, se hace sumamente difícil establecer especificaciones precisas para su elaboración que permitan estandarizar su calidad. Contrariamente a cuanto acontece con los automóviles y los discos compactos, en el transporte aéreo y los ejercicios aeróbicos los resultados no pueden ser medidos, comprobados y verificados para asegurar su calidad antes de la venta o entrega. Más aún, en el sector público es una simple prestación, los criterios que utilizan los ciudadanos para evaluarla pueden ser muy complejos y difíciles de establecer con precisión.

Segundo, los servicios (principalmente los que requieren mucha colaboración humana) son heterogéneos: por lo general, la prestación varía de un servicio a otro, de un ciudadano a otro y de un día a otro. La calidad de la interacción que el personal de atención ciudadana o las secretarias establecen con los ciudadanos muy raras veces puede ser estandarizada dentro de normas que permitan asegurar su calidad, como puede hacerse en una planta de productos manufacturados (Zeithaml, Parasuraman, y Berry, 1993, p. 17).

Tercero, la producción y el consumo de muchos servicios son inseparables. En términos generales, la calidad de los servicios se produce durante su entrega o prestación (usualmente como fruto de la interacción entre ciudadano y gobierno en el caso del sector público), en vez de ser estructurada y controlada en la planta de producción, lo que permite que el producto obtenido se entregue sin alteraciones al consumidor. A diferencia de los productores de bienes físicos, los proveedores de servicios no tienen el beneficio que les aporta toda una estructura que actúa como colchón amortiguador y que suaviza y matiza la relación entre la producción y el consumo. Con frecuencia, los ciudadanos se encuentran allí donde los servicios se producen, observando y evaluando el proceso de producción a medida que experimentan el servicio (Zeithaml, Parasuraman, y Berry, 1993, p. 18).

El resultado de acuerdo a lo que el personal de la D. A. P. Z cree que el ciudadano considera en la evaluación de la calidad en el servicio son en orden de importancia las siguientes (ver Tabla 3): 
Revista Eletrônica de Ciência Administrativa (RECADM) - ISSN 1677-7387

Faculdade Cenecista de Campo Largo - Coordenação do Curso de Administração

v. 2, n. 1, maio/2003 - http://revistas.facecla.com.br/index.php/recadm/

1. La habilidad del personal de la D. A. P. Z para realizar el servicio prometido en forma segura y precisa.

2. El conocimiento y trato amable del personal de la Dirección de Alumbrado Público de Zapopan y su forma de transmitir un sentimiento de confianza.

3. La disposición del personal de la Dirección de Alumbrado Público de Zapopan que muestra para ayudar a los clientes y darles un servicio rápido.

4. El cuidado y atención individualizada que la D. A. P. Z le da a sus clientes.

5. La apariencia de las instalaciones físicas, equipos, personal y material que utiliza la D. A. P. Z.

Tabla 3: Importancia de las dimensiones de calidad del servicio

Puntuaciones

\begin{tabular}{|l||c||c||c||}
\hline 20. Apariencia & 27 & 16.37 & 8.13 \\
\hline 21. Habilidad & 27 & 25.37 & 12.08 \\
\hline 22. Disposición & 27 & 19.07 & 9.20 \\
\hline $\begin{array}{l}\text { 23. Conocimiento y } \\
\text { trato amable }\end{array}$ & 27 & 22.22 & 10.95 \\
\hline $\begin{array}{l}\text { 24. Cuidado y } \\
\text { atención } \\
\text { individualizada }\end{array}$ & 27 & 16.96 & 8.98 \\
\hline
\end{tabular}

Del total de la muestra de este estudio, el $44.4 \%$ de las respuestas de los entrevistados concordó que la habilidad es la primera más importante de las cinco características mostradas anteriormente. El 37\% de las respuestas de los entrevistados coincidieron que la segunda característica más importante es la disposición de los sujetos, y por último la tercera característica más importante según el $51.9 \%$ fue la apariencia (ver Tablas 4, 5, y 6 ).

Tablas de Frecuencias

Tabla 4: Primera Más Importante

\begin{tabular}{|c|c|c|c|c|c|}
\hline & Frecuencia & $\begin{array}{l}\text { Porcenta } \\
\text { je }\end{array}$ & $\begin{array}{l}\text { Porcentaj } \\
\text { e Valido }\end{array}$ & $\begin{array}{c}\text { Porcentaj } \\
\text { e } \\
\text { Cumulativ } \\
0\end{array}$ \\
\hline \multirow{6}{*}{$\begin{array}{l}\text { Valid } \\
0\end{array}$} & Apariencia & 2 & 7.4 & 7.4 & 7.4 \\
\hline & Habilidad & 12 & 44.4 & 44.4 & 51.9 \\
\hline & Disposición & 5 & 18.5 & 18.5 & 70.4 \\
\hline & $\begin{array}{l}\text { Conocimien } \\
\text { to y trato } \\
\text { amable }\end{array}$ & 6 & 22.2 & 22.2 & 92.6 \\
\hline & $\begin{array}{l}\text { Cuidado y } \\
\text { atención } \\
\text { individualiz } \\
\text { ada }\end{array}$ & 2 & 7.4 & 7.4 & 100.0 \\
\hline & Total & 27 & 100.0 & 100.0 & \\
\hline
\end{tabular}

Tabla 5: Segunda Más Importante

\begin{tabular}{||c||c|c|c||c||}
\hline & $\begin{array}{c}\text { Frecuenci } \\
\text { a }\end{array}$ & $\begin{array}{c}\text { Porcentaj } \\
\text { e }\end{array}$ & $\begin{array}{c}\text { Porcentaj } \\
\text { e Valido }\end{array}$ & $\begin{array}{c}\text { Porcentaj } \\
e \\
\text { Cumulativ } \\
0\end{array}$ \\
\hline
\end{tabular}


Revista Eletrônica de Ciência Administrativa (RECADM) - ISSN 1677-7387

Faculdade Cenecista de Campo Largo - Coordenação do Curso de Administração v. 2, n. 1, maio/2003 - http://revistas.facecla.com.br/index.php/recadm/

\begin{tabular}{|c|c|c|c|c|c|}
\hline \multirow{6}{*}{$\begin{array}{l}\text { Valid } \\
\text { o }\end{array}$} & Apariencia & 2 & 7.4 & 7.4 & 7.4 \\
\hline & Habilidad & 5 & 18.5 & 18.5 & 25.9 \\
\hline & Disposición & 10 & 37.0 & 37.0 & 63.0 \\
\hline & $\begin{array}{l}\text { Conocimient } \\
\text { o y trato } \\
\text { amable }\end{array}$ & 7 & 25.9 & 25.9 & 88.9 \\
\hline & $\begin{array}{l}\text { Cuidado y } \\
\text { atención } \\
\text { individualizad } \\
\text { a }\end{array}$ & 3 & 11.1 & 11.1 & 100.0 \\
\hline & Total & 27 & 100.0 & 100.0 & \\
\hline
\end{tabular}

Tabla 6: Tercera Más Importante

\begin{tabular}{|c|c|c|c|c|c|}
\hline & $\begin{array}{c}\text { Frecuenci } \\
\text { a }\end{array}$ & $\begin{array}{c}\text { Porcentaj } \\
\text { e }\end{array}$ & $\begin{array}{l}\text { Porcentaj } \\
\text { e Valido }\end{array}$ & $\begin{array}{c}\text { Porcentaj } \\
\text { e } \\
\text { Cumulativ } \\
0\end{array}$ \\
\hline \multirow{6}{*}{$\begin{array}{l}\text { Valid } \\
\text { o }\end{array}$} & Apariencia & 14 & 51.9 & 51.9 & 51.9 \\
\hline & Habilidad & 1 & 3.7 & 3.7 & 55.6 \\
\hline & Disposición & 3 & 11.1 & 11.1 & 66.7 \\
\hline & $\begin{array}{l}\text { Conocimient } \\
\text { o y trato } \\
\text { amable }\end{array}$ & 3 & 11.1 & 11.1 & 77.8 \\
\hline & $\begin{array}{l}\text { Cuidado y } \\
\text { atención } \\
\text { individualizad } \\
\text { a }\end{array}$ & 6 & 22.2 & 22.2 & 100.0 \\
\hline & Total & 27 & 100.0 & 100.0 & \\
\hline
\end{tabular}

\section{Cumplimiento de las normas de Calidad en el Servicio (pregunta 3).}

La comprensión de las expectativas de los clientes constituye el primer paso en la realización de un servicio de alta calidad. Ahora bien, una vez que los directivos comprenden con exactitud lo que esperan los ciudadanos, deben afrontar un segundo reto, tan importante como el anterior: utilizar esos conocimientos para establecer en sus organizaciones las normas o estándares de calidad de los servicios.

Las normas señalan al personal de contacto cuáles son las prioridades de la dirección y cuál es el tipo de prestación que realmente cuenta. Cuando no existen normas estándar para la prestación o cuando las normas que se aplican no reflejan las expectativas del ciudadano o usuario (por ejemplo, cuando a los operadores del servicio de información de las empresas telefónicas se les evalúa sólo en función del número de llamadas que atienden al día) la calidad del servicio es percibida por los usuarios como algo que deben sufrir. Por el contrario, cuando existen normas que reflejan lo que los usuarios esperan (por ejemplo, trato amable, respuestas rápidas y promesas cumplidas) es muy probable que la percepción de calidad se incremente (Zeithaml, Parasuraman, y Berry, 1993, p. 43). A continuación se presentan los resultados obtenidos en este apartado.

En general, de las cinco características anteriores, el personal de la Dirección de Alumbrado Público de Zapopan respondió (variables 28 a 32) que son capaces de cumplir las normas formales o informales que existen para cada una de las dimensiones objeto de este estudio. 
El promedio más alto obtenido en cuanto a cumplimiento es para las normas relativas al grado de conocimiento y amabilidad que debe tener el personal de la D. A. P. $Z$ y el cuidado y la atención individualizada que el personal debe ofrecer a los usuarios. De forma contraria existe un mayor incumplimiento para las normas relativas a la apariencia, la habilidad y la disposición. (Ver Tabla 7).

Tabla 7: Cumplimiento de las normas de calidad

Stad.

\begin{tabular}{|c|c|c|c|}
\hline $\begin{array}{l}\text { 28. Normas de } \\
\text { apariencia }\end{array}$ & 27 & 7.67 & 2.00 \\
\hline 29. Normas de habilidad & 27 & 7.74 & 2.33 \\
\hline $\begin{array}{l}\text { 30. Normas de } \\
\text { disposición }\end{array}$ & 27 & 7.74 & 2.30 \\
\hline $\begin{array}{l}\text { 31. Normas de } \\
\text { conocimiento y } \\
\text { amabilidad }\end{array}$ & 27 & 8.19 & 1.98 \\
\hline $\begin{array}{l}\text { 32. Normas de cuidado y } \\
\text { la atención } \\
\text { individualizada }\end{array}$ & 27 & 8.07 & 2.11 \\
\hline
\end{tabular}

\section{Cumplimiento de promesas sobre el servicio (pregunta 4).}

Un factor clave y determinante en la formación de las expectativas del ciudadano o usuario es la comunicación externa de los proveedores del servicio. Las promesas que hace una dirección o empresa de servicios en la publicidad que realiza en los medios de comunicación, en los mensajes que transmite y en otras comunicaciones similares, incrementan las expectativas. Estas a su vez, actúan como las normas contra las que los clientes evaluarán lo que consideran un servicio de calidad. Una discrepancia entre el servicio real percibido y las promesas de servicio tienen, por lo tanto, un efecto negativo sobre la percepción de calidad del servicio (Zeithaml, Parasuraman, y Berry, 1993, p. 49).

La posibilidad de que se prometa en exceso es muy alta, debido a que la comunicación de las empresas u organizaciones de servicio promete lo que hacen las personas $\mathrm{y}$, dado que a las personas no se pueden controlar de la misma forma en que se pueden controlar las máquinas que producen buenos productos, el potencial para que se produzcan promesas poco realistas es muy alto.

Para el personal de la Dirección De Alumbrado Público de Zapopan las promesas relativas al conocimiento y amabilidad, y el cuidado y la atención individualizada con los usuarios son las que se cumplen. Contrariamente las promesas de apariencia, habilidad en su prestación y dispuesto a atender los problemas de la sociedad, son las dimensiones en las que, en menor medida, se cumplen promesas quizás porque no se hace énfasis en ellas (variables 33 a 37). (Ver Tabla 8).

\section{Tabla 8: Cumplimiento de promesas}

\begin{tabular}{|l|c|c||c||}
\multicolumn{1}{|l|}{} & N & Media & Desv. Stad \\
\hline 33. Cumplir apariencia & 27 & 7.33 & 2.09 \\
\hline 34. Cumplir habilidad & 27 & 7.63 & 2.11 \\
\hline $\begin{array}{l}\text { 35. Cumplir } \\
\text { disposición }\end{array}$ & 27 & 7.70 & 2.05 \\
\hline $\begin{array}{l}\text { 36. Cumplir } \\
\text { conocimiento y } \\
\text { amabilidad }\end{array}$ & 27 & 8.41 & 1.15 \\
\hline 37. Cumblir cuidado v & 27 & 8.70 & 1.35 \\
\hline
\end{tabular}


Revista Eletrônica de Ciência Administrativa (RECADM) - ISSN 1677-7387

Faculdade Cenecista de Campo Largo - Coordenação do Curso de Administração

v. 2, n. 1, maio/2003 - http://revistas.facecla.com.br/index.php/recadm/

\section{la atención \\ individualizada}

\section{Aspectos organizacionales para la prestación del servicio y la contribución del} personal interno a la calidad en el servicio (preguntas 5 y 6 ).

Los antecedentes potenciales relacionados con aspectos organizacionales y culturales dentro de esta área correspondientes a la discrepancia entre las normas de calidad del servicio y el servicio real y la discrepancia entre el servicio real y lo que se comunica a los ciudadanos sobre él muestran que el trabajo en equipo, el entendimiento entre el empleado y sus funciones, el entendimiento entre la tecnología y su función y el control percibido dentro de la organización son aceptables sin llegar a lo óptimo.

En cuanto a los sistemas de supervisión y control el personal se muestra confundido respecto a que se le da mayor valor dentro de sus evaluaciones, sí el trato con el ciudadano o el desempeño de sus funciones. Con relación a la comunicación horizontal los resultados obtenidos muestran poca eficacia dado que el personal se muestra confundido en torno a que servicios que ofrece esta área (ver Tabla 9 y 10).

Tabla 9: Cumplimiento de aspectos organizacionales

\begin{tabular}{|c|c|c|c|}
\hline & $\mathbf{N}$ & Media & Desv. Stad. \\
\hline $\begin{array}{l}\text { 38. Recopilar } \\
\text { información sobre } \\
\text { usuarios }\end{array}$ & 27 & 7.59 & 2.53 \\
\hline $\begin{array}{l}\text { 39. Utilizar } \\
\text { investigaciones de } \\
\text { usuarios }\end{array}$ & 27 & 5.00 & 3.25 \\
\hline $\begin{array}{l}\text { 40. Información sobre } \\
\text { lo que esperan los } \\
\text { ciudadanos }\end{array}$ & 27 & 7.81 & 2.68 \\
\hline $\begin{array}{l}\text { 41. Interacción de los } \\
\text { superiores con los } \\
\text { usuarios }\end{array}$ & 27 & 5.89 & 3.63 \\
\hline $\begin{array}{l}\text { 42. Comunicación con } \\
\text { los superiores }\end{array}$ & 27 & 7.41 & 2.50 \\
\hline $\begin{array}{l}\text { 43. Sugerencias del } \\
\text { personal a los } \\
\text { superiores }\end{array}$ & 27 & 5.30 & 3.29 \\
\hline $\begin{array}{l}\text { 44. Interacción } \\
\text { superiores con el } \\
\text { personal }\end{array}$ & 27 & 7.48 & 2.68 \\
\hline 45. Niveles jerárquicos & 27 & 7.04 & 3.04 \\
\hline $\begin{array}{l}\text { 46. Asignación de } \\
\text { recursos }\end{array}$ & 27 & 5.22 & 3.68 \\
\hline $\begin{array}{l}\text { 47. Programas } \\
\text { internos de servicio a } \\
\text { usuarios }\end{array}$ & 27 & 7.81 & 2.82 \\
\hline $\begin{array}{l}\text { 48. Estimulación a } \\
\text { dirigentes }\end{array}$ & 27 & 6.07 & 3.25 \\
\hline $\begin{array}{l}\text { 49. Proceso formal de } \\
\text { objetivos de calidad }\end{array}$ & 27 & 6.74 & 3.35 \\
\hline $\begin{array}{l}\text { 50. Establecer } \\
\text { objetivos específicos } \\
\text { de calidad }\end{array}$ & 27 & 8.22 & 2.39 \\
\hline 51. Automatización en & 27 & 7.63 & 2.31 \\
\hline
\end{tabular}


Revista Eletrônica de Ciência Administrativa (RECADM) - ISSN 1677-7387

Faculdade Cenecista de Campo Largo - Coordenação do Curso de Administração v. 2, n. 1, maio/2003 - http://revistas.facecla.com.br/index.php/recadm/

\begin{tabular}{|c|c|c|c|}
\hline el servicio & & & \\
\hline $\begin{array}{l}\text { 52. Programas de } \\
\text { mejora }\end{array}$ & 27 & 6.63 & 3.16 \\
\hline $\begin{array}{l}\text { 53. Conocimiento de } \\
\text { requerimientos del } \\
\text { usuario }\end{array}$ & 27 & 7.19 & 2.80 \\
\hline $\begin{array}{l}\text { 54. Sistemas } \\
\text { operativos de } \\
\text { computación }\end{array}$ & 27 & 6.81 & 2.86 \\
\hline
\end{tabular}

Tabla 10: Aspectos culturales de la organización

\begin{tabular}{|c|c|c|c|}
\hline & $\mathbf{N}$ & Media & Desv. Stad. \\
\hline 55. Parte de un equipo & 27 & 8.93 & 2.18 \\
\hline $\begin{array}{l}\text { 56. Todos contribuyen al } \\
\text { esfuerzo }\end{array}$ & 27 & 8.41 & 2.48 \\
\hline 57. Responsable de ayudar & 27 & 9.37 & 1.21 \\
\hline $\begin{array}{l}\text { 58. Cooperación entre los } \\
\text { compañeros }\end{array}$ & 27 & 8.48 & 2.38 \\
\hline $\begin{array}{l}\text { 59. Miembro importante de la } \\
\text { dirección }\end{array}$ & 27 & 8.33 & 2.24 \\
\hline 60. Capaz de realizarlo bien & 27 & 9.30 & 1.75 \\
\hline 61. Personal calificado & 27 & 7.04 & 3.22 \\
\hline $\begin{array}{l}\text { 62. Instrumentos y equipo } \\
\text { necesario }\end{array}$ & 27 & 7.56 & 2.56 \\
\hline $\begin{array}{l}\text { 63. Resolver problemas con } \\
\text { poco control }\end{array}$ & 27 & 5.56 & 3.36 \\
\hline $\begin{array}{l}\text { 64. Libertad de satisfacer las } \\
\text { necesidades de los usuarios }\end{array}$ & 27 & 7.48 & 2.99 \\
\hline $\begin{array}{l}\text { 65. No tengo control sobre mi } \\
\text { trabajo }\end{array}$ & 27 & 5.81 & 3.29 \\
\hline 66. Depender de otros & 27 & 5.96 & 3.56 \\
\hline $\begin{array}{l}\text { 67. Evaluaciones incluyen } \\
\text { interacción con usuarios }\end{array}$ & 27 & 6.96 & 3.02 \\
\hline $\begin{array}{l}\text { 68. Mayor esfuerzo no implica } \\
\text { mayor pago }\end{array}$ & 27 & 6.93 & 3.36 \\
\hline $\begin{array}{l}\text { 69. Cantidad de papeleo dificulta } \\
\text { el servicio }\end{array}$ & 27 & 5.59 & 3.35 \\
\hline 70. Clientes y jefes es lo mismo & 27 & 7.26 & 3.02 \\
\hline $\begin{array}{l}\text { 71. La dirección y yo tenemos las } \\
\text { mismas ideas }\end{array}$ & 27 & 7.04 & 2.81 \\
\hline 72. Información sobre mi trabajo & 27 & 7.33 & 2.97 \\
\hline $\begin{array}{l}\text { 73. No entiendo los servicios que } \\
\text { ofrecemos }\end{array}$ & 27 & 3.74 & 3.45 \\
\hline $\begin{array}{l}\text { 74. Capaz de adecuarme a los } \\
\text { cambios }\end{array}$ & 27 & 8.81 & 1.94 \\
\hline 75. Entrenamiento suficiente & 27 & 3.81 & 3.49 \\
\hline $\begin{array}{l}\text { 76. No sé que aspectos que se le } \\
\text { da mayor importancia }\end{array}$ & 27 & 5.26 & 3.41 \\
\hline $\begin{array}{l}\text { 77. Consultan trabajadores sobre } \\
\text { la veracidad de las promesas }\end{array}$ & 27 & 5.74 & 3.16 \\
\hline
\end{tabular}


Revista Eletrônica de Ciência Administrativa (RECADM) - ISSN 1677-7387

Faculdade Cenecista de Campo Largo - Coordenação do Curso de Administração

v. 2, n. 1, maio/2003 - http://revistas.facecla.com.br/index.php/recadm/

\begin{tabular}{|l||c||c||c||}
\hline $\begin{array}{l}\text { 78. Interacción con el personal } \\
\text { directo al servicio }\end{array}$ & 27 & 5.30 & 3.30 \\
\hline $\begin{array}{l}\text { 79. La política es la misma en las } \\
\text { diferentes oficinas }\end{array}$ & 27 & 6.41 & 3.82 \\
\hline Validos. & 27 & & \\
\hline
\end{tabular}

\section{Conclusiones.}

La medición de la calidad en los servicios públicos a través del modelo SERVQUAL, nos permite conocer la importancia dada a las dimensiones de la calidad del servicio y detectar las deficiencias en el servicio.

Los hallazgos que surjan de estos estudios o mediciones, analizados en conjunto, sugieren la existencia de algunas oportunidades clave, para muchas organizaciones, de perfeccionar los niveles de la calidad percibida en sus servicios. Esos hallazgos también muestran los significativos beneficios que las organizaciones públicas pueden obtener al proporcionar servicios de una calidad superior. En el caso de las organizaciones que proveen cualquier tipo de servicios la clave para facilitar un servicio de alta calidad radica en equilibrar las expectativas y las percepciones de los usuarios o acabar con las diferencias o desequilibrios que pudiesen existir entre ambas.

Dentro del modelo SERVQUAL se identifican cuatro factores clave causales de las deficiencias internas que contribuyen a los bajos niveles de calidad en el servicio percibido por los usuarios; es decir: Deficiencia 1, 2, 3, 4 y 5. Este modelo nos permitió la comprensión y medición de la calidad en el servicio de esta dirección, así como también le permitirá a esta área el perfeccionamiento interno de la calidad en el servicio.

Es importante mencionar que en algunos otros estudios de medición de la calidad en los servicios se mostró que la fiabilidad es la dimensión más importante y la dimensión respecto a los elementos tangibles es el menos importante, como se puede comprobar, en esta investigación se obtuvieron los mismos resultados.

Para llevar a cabo todas las funciones del servicio de calidad esta área cuenta con programas internos para la mejora de la calidad en el servicio, recordemos que la D. A. P. Z. cuenta con la certificación ISO 9002 y próximamente obtendrá la certificación ISO 9000:2000 que está permitirá una aplicación más adecuada para las organizaciones públicas, así como un mayor reconocimiento entre la organización y el ciudadano.

\section{Recomendaciones.}

Reconociendo en esta investigación los avances de la filosofía de calidad en el servicio por parte de la dirección y del personal de la Dirección de Alumbrado Público de Zapopan se presentan algunas recomendaciones respecto a los aspectos que se pudieran considerarse vulnerables en la aplicación o seguimiento de la calidad en el servicio de esta área o de alguna otra área de las mismas características.

Es importante el establecimiento de normas de calidad para el aseguramiento de un nivel alto de servicio al ciudadano. Por esto, la realización de un servicio de calidad requiere un fuerte liderazgo y compromiso por parte de la alta dirección. Sin ese compromiso y disposición para aceptar las dificultades temporales que implica todo cambio, la calidad en el servicio sencillamente no se produce. Contactar con el personal y directivos de mandos medios no mejora ni puede mejorar la calidad si no existe un fuerte liderazgo por parte de la alta dirección.

Finalmente, para tener una visión completa y equilibrar las percepciones del personal y dirección del área de alumbrado público del Ayuntamiento de Zapopan y las expectativas de los ciudadanos que reciben este servicio, es importante realizar un estudio similar a esta investigación teniendo como objetivo principal la opinión de la ciudadanía. 
Revista Eletrônica de Ciência Administrativa (RECADM) - ISSN 1677-7387

Faculdade Cenecista de Campo Largo - Coordenação do Curso de Administração

v. 2, n. 1, maio/2003 - http://revistas.facecla.com.br/index.php/recadm/

\section{Bibliografía.}

Bowman, J. S. , \& French, B. J. (1992, Fall). Quality Improvement in a State Agency Revisited. Public Productivity \& Management Review, 16 (1), 56 - 58.

De la Cerda, J. , \& Morales, L. (2001). Calidad ISO 9000 En La Administración Pública De México. Grupo Editorial Iberoamérica.

Dobbs, M. F. (1994, Fall). Continuous Improvement as Continuous Implementation: Implementing TQM in the City of Santa Ana. Public Productivity \& Management Review, $18(1), 89-92$.

Hyde, A. C. (1992, Fall). Implications of Total Quality Management for the Public Sector. Public Productivity \& Management Review, 16 (1), 23, 24, 25 - 36.

Kravchuck, R. S. , \& Leighton, R. (1993, Fall). Implementing Total Quality Management in the United States. Public Productivity \& Management Review, 17, 71- 83.

Milakovich, M. E. (1990, Fall). Total Quality Management for Public Sector Productivity Improvement. Public Productivity \& Management Review, 14 (1), 19-32.

Moon, C. , \& Swaffin-Smith, C. (May, 1998). Total Quality Management and New Patterns of Work: Is There Life Beyond Empowerment?. Total Quality Management, 19 (2/3), 301310.

Nwabueze, U. , \& Kanji, G. J. (1997) The Implementation of Total Quality Management in the NHS: How to Avoid Failure. Total Quality Management, 8 (5), 265-270.

Pike, J. \& Barnes, R. (1996) TQM in Action (London, Chapman \& Hall).

Sureschchandar, G. S. \& Rajendran, C. , \& Anantharaman, R. N. (May, 2001). A Conceptual Model for Total Quality Management in Service Organizations. Total Quality Management, 12 (3), 343-360.

West, J. P. , \& Berman, E. M. , \& Milakovich M. E. (1993, Winter). Implementing TQM in Local Government: The Leadership Challenge. Public Productivity \& Management Review, 17 (2), 178 - 180, $184-186$.

Zeithaml, V. A. , Parasuraman, A. , \& Berry, L. (1993). Calidad Total En La Gestión de Servicios: Cómo lograr el equilibrio entre las percepciones y las expectativas de los consumidores. Díaz de Santos, S.A. 